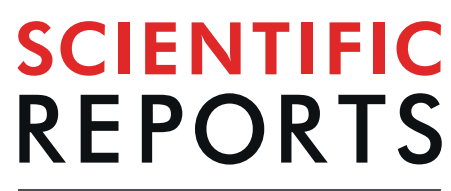

natureresearch

\title{
Downregulation of histone methyltransferase SET8 inhibits progression of hepatocellular carcinoma
}

\author{
Jianhua Wu $u^{1,5}$, Kuangyuan Qiao ${ }^{4,5}$, Yanming Du² ${ }^{2}$ Xiaoyun Zhang ${ }^{2}$, Haichao Cheng ${ }^{3}$, Li Peng ${ }^{3 *}$ \\ \& Zhanjun Guo ${ }^{2 *}$ \\ The expression of lysine methyltransferase SET8, which is involved in carcinogenesis of many types \\ of human cancers through monomethylation of histone $\mathrm{H} 4$ lysine 20 (H4K20), is associated with the \\ prognosis of hepatocellular carcinoma (HCC). We performed a functional analysis for SET8 to assess its \\ effect on HCC progression. SET8 knockdown inhibited proliferation, migration and invasion of HCC cells. \\ SET8 knockdown also inhibited tumour growth in a human xenograft mouse model. Overexpression of \\ SET8 displayed the reverse effect, while treatment with the SET8 inhibitor UNC0379 produced an effect \\ similar to SET8 knockdown. In addition, drug sensitivity testing in SET8-siRNA transfected HCC cells \\ indicated that docetaxel inhibited cell growth dramatically, as demonstrated by the Cell Counting Kit-8 \\ (CCK-8) assay. Furthermore, gene expression microarray analysis showed that genes altered after SET8 \\ knockdown were clustered in pathways related to tumorigenesis and metastasis. Our data suggests \\ that targeting SET8 for HCC therapy can inhibit the proliferation and invasion of HCC cells as well as \\ increase their sensitivity to chemotherapy.
}

Hepatocellular carcinoma (HCC) is a highly aggressive malignant tumour that accounted for approximately 782,500 new liver cancer cases and 745,500 deaths worldwide in $2012^{1}$. Despite significant developments in the diagnosis and treatment of HCC, the overall survival of patients with HCC remains low due to distant metastasis, local recurrence, treatment resistance, and lack of early diagnosis. Molecular targets underlying the mechanisms of HCC development, progression and metastasis may provide novel effective therapeutic targets for HCC treatment ${ }^{2}$.

Post-translational histone modifications, including methylation, acetylation, phosphorylation, ubiquitination, and ADP-ribosylation, are involved in a number of cellular processes such as mitosis, meiosis, DNA replication, and the DNA damage response ${ }^{3}$. Histone $\mathrm{H} 4$ methylation is one of the most intriguing histone modifications with the majority of histone $\mathrm{H} 4$ methylation occurring in the $\mathrm{N}$-terminal tail of lysine 20 (H4K20). PR-Set7/Set8/ KMT5a (SET8) encodes an H4K20 monomethyltransferase that is implicated in normal cell cycle progression including DNA replication, DNA damage repair, and cell cycle control ${ }^{4,5}$. SET8 could exert specific effects at the origin of DNA replication through interacting with the DNA replication factor proliferating cell nuclear antigen (PCNA), and SET8 depletion causes cells to arrest in the S phase with increased DNA damage and apoptosis ${ }^{6-10}$. Additionally, SET8 binds and methylates non-histone oncoproteins such as p53, TWIST, Wnt, estrogen receptor alpha $(\mathrm{ER} \alpha)$ and androgen receptor $(\mathrm{AR})$, and recruits p53BP1 during the DNA double-strands break response ${ }^{11-14}$. Furthermore, SET8 mediates the dual transcriptional activities of TWIST and promotes epithelial-mesenchymal transition leading to increased metastatic capacity of breast cancer cells ${ }^{12,15}$.

We have previously identified that altered SET8 expression modified HCC outcome ${ }^{16}$. Here, we performed a functional analysis for SET8 to assess its effect on HCC progression.

\footnotetext{
${ }^{1}$ Animal center, The Fourth Hospital of Hebei Medical University, Shijiazhuang, P.R. China. ${ }^{2}$ Department of Rheumatology and Immuology, The Fourth Hospital of Hebei Medical University, Shijiazhuang, P.R. China. ${ }^{3}$ Department of Hepatobiliary Surgery, The Fourth Hospital of Hebei Medical University, Shijiazhuang, P.R. China. ${ }^{4}$ Basic Medical College, Hebei Medical University, Shijiazhuang, P.R. China. ${ }^{5}$ These authors contributed equally: Jianhua Wu and Kuangyuan Qiao. *email: pengli72@sina.com; zjguo5886@aliyun.com
} 


\begin{tabular}{|l|l|}
\hline Name & Sequence \\
\hline SET8-siRNA1 & CAGAATCGCAAACTTACGGAT \\
\hline SET8-siRNA2 & GAATGAAGATTGACCTCATCG \\
\hline SET8-siRNA3 & GCCTAGGAAGACTGATCAATC \\
\hline SET8-siRNA4 & GGCGCTCACTGAAGTGTATGA \\
\hline & ATGGCTAGAGGCAGGAAGATGTCCAAGCCCCGCGCGGTGGA \\
& GGCGGCGGCGGCGGCGGCGGCGGTGGCAGCGACGGCCCCGG \\
& GCCCGGAGATGGTGGAGCGGAGGGGCCCGGGGAGGCCCCGCACCGACGGGGAGAACGT \\
& ATTTACCGGGCAGTCAAAGATCTATTCCTACATGAGCCCGAACAAATGCTCTG \\
& GAATGCGTTCCCCTTCAGGAAGAGAACTCAGTTACACATCACGAAGTCAA \\
& ATGCCAGGGGAACCATTAGCCGGAATCTACAGGAAACGAGAAGAGAAAAG \\
& AAATGCTGGGAACGCAGTACGGAGCGCCATGAAGTCCGAGGAACAGAAGAT \\
& CAAAGACGCCAGGAAAGGTCCCCTGGTACCTTTTCCAAACCAAAAATCTGAAGCAGC \\
& AGAACCTCCAAAAACTCCACCCTCATCTTGTGATTCCACCAATGCAG \\
& CCATCGCCAAGCAAGCCCTGAAAAAGCCCATCAAGGGCAAACAGGCC \\
ORF nucleotide sequence of SET8 & CCCGGAAAAAAAGCTCAAGGAAAAACGCAACAGAATCGCAAACTTAC \\
& GGATTTCTACCCTGTCCGAAGGAGCTCCAGGAAGAGCAAAGCCGAGC \\
& TGCAGTCTGAAGAAAGGAAAAGAATAGATGAATTGATTGAAAGTGGG \\
& AAGGAAGAAGGAATGAAGATTGACCTCATCGATGGCAAAGGCAGGGG \\
& TGTGATTGCCACCAAGCAGTTCTCCCGGGGTGACTTGTGGTGGAT \\
& ACCACGGGGACCTCATCGAGATCACCGACGCCAAGAAACGGGAGGCT \\
& CTGTACGCACAGGACCCTTCCACGGGCTGCTACATGTACTATTTTCA \\
& GTATCTGAGCAAAACCTACTGCGTGGATGCAACTAGAGAGACAAATC \\
& GCCTAGGAAGACTGATCAATCACAGCAAATGTGGGAACTGCCAAACC \\
& AAACTGCACGACATCGACGGCGTACCTCACCTCATCCTCATCGCCTC \\
& CCGAGACATCGCGGCTGGGGAGGAGCTCCTGTATGACTATGGGGACC \\
& GCAGCAAGGCTTCCATTGAAGCCCACCCGTGGCTGAAGCATTAG \\
\hline
\end{tabular}

Table 1. The sequence of siRNA and cDNA clone of SET8.

\section{Methods}

Cell culture and transfection. Six human hepatic carcinoma cell lines (QGY-7701, Hepg2, LM3, Huh-7, SMMC-7721, Hep3B) were purchased from the Shanghai Institute of Biological Sciences, Chinese Academy of Sciences (Shanghai, China) and cultured in DMEM/RPMI-1640 medium (Gibco ${ }^{\text {TM }}$ Life Technologies, Grand Island, NY) supplemented with $10 \%$ foetal bovine serum (FBS) (Gibco ${ }^{\mathrm{TM}}$ Life Technologies, Grand Island, NY) in a humidified incubator containing $5 \% \mathrm{CO}_{2}$ at $37^{\circ} \mathrm{C}$.

SMMC-7721 cells in the logarithmic growth phase were seeded into 6-well plates. psi-H1-SET8siRNA and psi-H1 plasmids expressing green fluorescent protein (GFP) (GeneCopoeia, Rockville, MD) were transfected into SMMC-7721 cells using Lipofactamine 2000 (Invitrogen, San Diego, CA). To overexpress SET8, the coding sequence of SET8 was amplified and subcloned into the pEZ-M61 vector, having a puromycin selection marker (GeneCopoeia). Huh-7 cells were then transfected with SET8-pEZ-M61 and pEZ-M61 plasmids using lipofectamine 2000 (Invitrogen). After $72 \mathrm{~h}$ of incubation, $0.6 \mu \mathrm{g} / \mathrm{ml}$ puromycin (Sigma-Aldrich, St. Louis, MO) was added into each well for 10-12 days and resistant clones were selected. The siRNA and cDNA clone sequences are listed in Table 1.

Western blot analysis. Total protein was extracted using radio immunoprecipitation assay (RIPA) lysis buffer with freshly added protease inhibitor (Roche, Basel, Switzerland). Western blot analysis was performed as described previously ${ }^{17}$. Briefly, $40 \mu \mathrm{g}$ total protein extract was loaded into a $10 \%$ sodium dodecyl sulphate polyacrylamide gel (SDS-PAGE) followed by transfer onto a polyvinylidene difluoride (PVDF) membranes (Roche, Basel, $\mathrm{CH}$ ). Membranes were blocked with $5 \%$ skim milk, incubated first with rabbit polyclonal antibodies against human SET8 (1:1000; Abcam, Cambridge, UK), p53 (1:2,000; Santa Cruz, CA), anti-p53K382me1(1:1000; Affinity Biosciences, Cincinnati, $\mathrm{OH})^{11}$, or $\beta$-actin $\left(1: 10,000\right.$; Santa Cruz, CA) overnight at $4{ }^{\circ} \mathrm{C}$, followed by incubation with secondary HRP-conjugated anti-rabbit IgG antibody (1:5000; Thermo Fisher, New York, NY). Proteins were visualized with an enhanced chemiluminescence reagent (Thermo Fisher, New York, NY) using the FluorChem ${ }^{\circledR}$ HD2 protein imprinting imaging system (Alpha InnoTec, San Leandro, CA).

Cell proliferation assay. Cell proliferation was examined using the Cell Counting Kit (CCK) - 8 (Dojindo Lab, Kumamoto, Japan). Briefly, cells stably transfected with psi-H1-SET8siRNA or SET8-pEZ-M61 in the logarithmic growth phase were seeded into 96 -well microplates with a density of $1 \times 10^{3}$ cells per well. Non-transfected SMMC-7721, Huh-7 and Hepg2 cells were also treated with the SET8 inhibitor UNC0379 (MCE, Monmouth Junction, NJ) for $72 \mathrm{~h}$. At each time point $(0,12,24,48,60$ and $72 \mathrm{~h}), 10 \mu \mathrm{l}$ of diluted CCK-8 was added into each well and cells were incubated in a $5 \%$ humidified $\mathrm{CO}_{2}$ incubator for $2 \mathrm{~h}$ at $37^{\circ} \mathrm{C}$. Absorbance was measured at $450 \mathrm{~nm}$ using a Microplate Autoreader (Bio-Tek; Instruments, VT).

Wound healing assay. Cells were cultured in 6-well plates. A scratch was created on the cell layer using a $200 \mu$ pipette tip when cells reached $100 \%$ confluence. Subsequently, cells were washed twice with PBS. Images of the cells in each group were captured at $0,12,24$ and $48 \mathrm{~h}$ after scratching. At least five fields were analysed for each scratch and cell migration rates were calculated using the following formula ${ }^{18}$. 


$$
\text { Percentage wound healing }=\frac{(\text { wound length at } 0 \mathrm{~h})-(\text { wound length at } 12,24 \mathrm{~h} \text { or } 48 \mathrm{~h})}{\text { wound length at } 0 \mathrm{~h}} \times 100 \%
$$

Each experiment was repeated three times.

Cell invasion assay. Cell invasion assays were performed using $8 \mu \mathrm{m}$ Transwell chambers containing polycarbonate filters (pore size, 8; Corning, NY) with Matrigel (BD Biosciences, NJ). Cells stably transfected with psi-H1-SET8siRNA or SET8-pEZ-M61 $\left(5 \times 10^{4}\right.$ cells per well) grown in $200 \mu \mathrm{l}$ serum-free medium were placed in the upper chambers; SMMC-7721 and Huh-7 cells treated with UNC0379 in $200 \mu l$ serum-free medium were also placed in the upper chambers. $500 \mu \mathrm{l}$ DMEM medium containing $15 \%$ FBS was added to the lower chamber, and cells were allowed to invade through the matrigel for $24 \mathrm{~h}$ at $37^{\circ} \mathrm{C}$. Cells which invaded into the underside of the membrane were fixed with $4 \%$ paraformaldehyde and stained with $0.1 \%$ crystal violet. Stained cells were counted under an inverted microscope (Nikon, Tokyo, Japan) in five randomly selected fields (magnification, x 200).

Flow cytometry. Cell apoptosis was assessed by flow cytometry analysis. SMMC-7721 cells were harvested and washed twice with cold PBS and once with binding buffer, and then incubated with PE-conjugated Annexin $\mathrm{V}$ and 7-AAD (BD Biosciences, Pharmingen, San Diego, CA) for 15 minutes in the dark at $25^{\circ} \mathrm{C}$. Cells were then resuspended in binding buffer and analyzed using a fluorescence-activated cell sorting (FACS) Aria II flow cytometer (BD Biosciences). Each experiment was repeated three times.

Nude mice for tumour cell xenograft assays. Twenty-four four-week-old female $B A L B / c$ nude mice were purchased from Charles River Laboratories [Beijing, China; permission no. SCXK (Jing) 2016-0006]. The mice were kept in a clean environment at the Laboratory Animal Centre in the Fourth Hospital of Hebei Medical University and provided standard rodent chow and water ad libitum. Animal experiments were conducted in accordance with the National Institutes of Health (USA) guidelines for the care and use of laboratory animals ${ }^{19}$ and approved by the Institutional Animal Care and Ethics Committee at The Fourth Hospital of Hebei Medical University.

SMMC-7721 cells $\left(5 \times 10^{6}\right.$ cells/mouse in $200 \mu \mathrm{l}$ DMEM) stably transfected with psi-H1-SET8siRNA or psi-H1 were subcutaneously injected in the left scapular region of each mouse. Tumour growth was monitored at regular intervals by measuring tumour diameter using a calliper. Tumour volume was determined using the following formula.

$$
\text { Tumour size }=\frac{\text { length } \times \text { width }^{2}}{2}
$$

In vivo green fluorescent imaging was performed using the NightOwl Bioimager (Berthold Technologies, Bad Wildbad, Germany) once a week. Signal intensity was quantified using the WinLight32 software package (Berthold Technologies).

Chemosensitivity assay. Drug sensitivity testing for the chemotherapy reagents cisplatin, docetaxel, 5-fluorouracil, and etoposide (TCI chemicals, Shanghai, China) was performed in SET8-siRNA transfected SMMC-7721 cells with the CCK-8 assay as described above. Cells were treated for $72 \mathrm{~h}$ prior to CKK-8 assay with the following: cisplatin, $0,0.2,0.4,0.6,0.8,1.0,1.2 \mathrm{mg} / \mathrm{ml}$; docetaxel, $0,20,40,60,80,100,120 \mathrm{mg} / \mathrm{ml}$; 5 -fluorouracil (5-Fu), 0, 0.3, 0.6, 0.9, 1.2, 1.5, $1.8 \mathrm{mg} / \mathrm{ml}$; etoposide, $0,0.2,0.4,0.6,0.8,1.0,1.2 \mathrm{mg} / \mathrm{ml}$.

Microarray analysis. The microarray experiment was commissioned to CapitalBio Corporation (Beijing, China). Briefly, SMMC-7721 cells stably transfected with psi-H1-SET8siRNA or psi-H1 were harvested and washed three times with PBS. Total RNA was extracted using TRIzol (Invitrogen, Carlsbad, CA). Subsequently, RNA samples were reverse-transcribed to generate cDNA. Three biological replicates for each group were used. Microarray manufacturing and processing were performed as described ${ }^{20}$. Microarrays were scanned on a GeneChip Scanner 3000 (Affymetrix, Santa Clara, CA), and data analysis was performed using GeneChip Operating Software (GCOS version 1.4, Affymetrix, Santa Clara, CA). Significantly altered genes following SET8 knockdown were investigated for biological processes and signalling pathways using the cytoscape plug-in of Reactome $\mathrm{FI}^{21}$.

Statistical analysis. Data are expressed as means \pm standard deviation. Statistical analysis between two groups was performed with Student's $t$ test, and the comparison between three or more groups was performed with One-way ANOVA. $\mathrm{P}<0.05$ was considered statistically significant. Statistical analyses were performed using SPSS 19.0 software package (SPSS Inc., Chicago, IL).

\section{Results}

Downregulation of SET8 inhibits proliferation, migration and invasion of HCC cells. SET8 protein expression was assayed in HCC cell lines including QGY-7701, Hepg2, LM3, Huh-7, SMMC-7721 and Hep3B cells with Western blotting (Fig. 1A). Since SMMC-7721 displayed high SET8 expression whereas Huh-7 displayed low SET8 expression, we use these two cell lines for subsequent knockdown and overexpression assays. Four psi-H1-SET8siRNAs were successfully transfected into SMMC-7721 cells. Western blot analysis indicated that SET8 levels significantly decreased in SET8 siRNA2 transfected cells compared with other SET8 siRNAs transfected cells (Fig. 1B); therefore, we used the SET8 siRNA2 construct for subsequent analysis. 
A

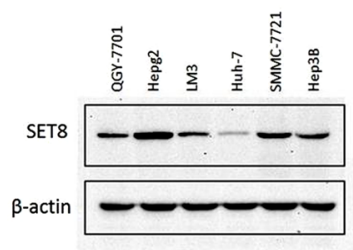

D

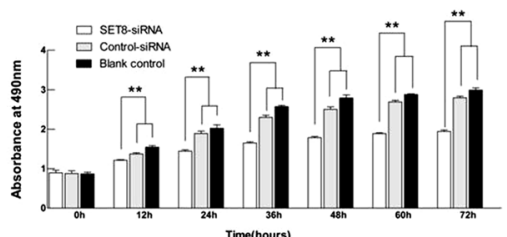

G

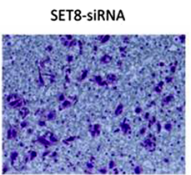

Blank-control
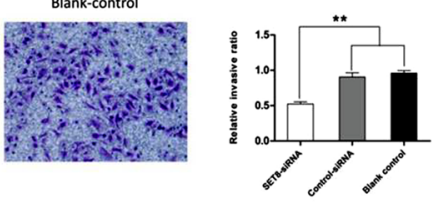

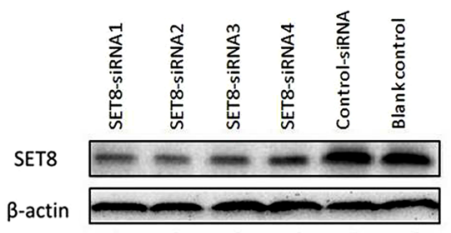

$\mathbf{E}$

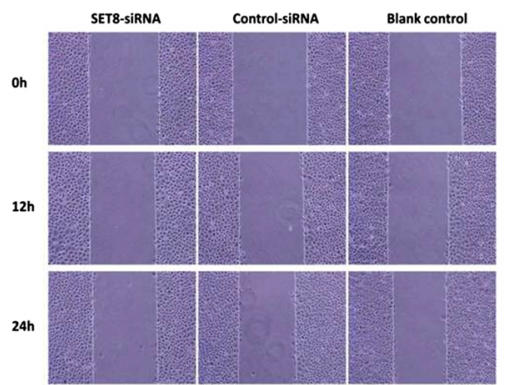

H

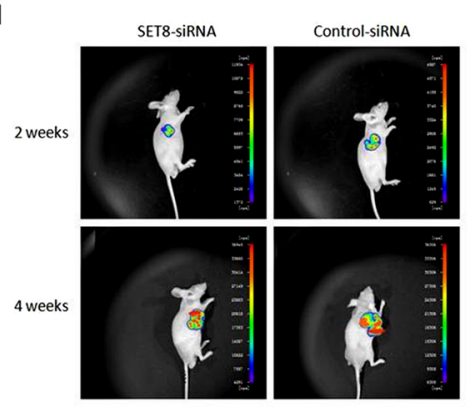

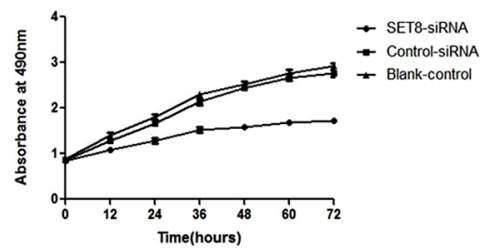

$\mathbf{F}$
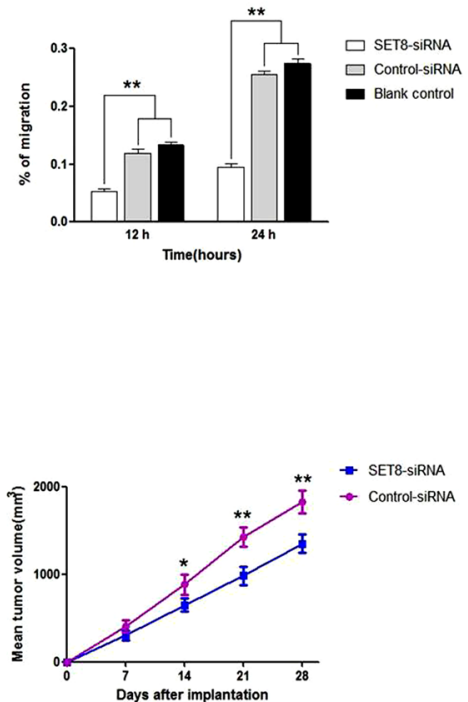

K

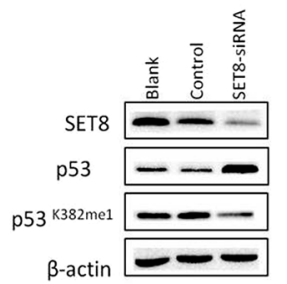

Figure 1. SET8 knockdown inhibits proliferation, migration and invasion of HCC cells and inhibits HCC cell growth in vivo. (A) Expression of SET8 protein levels was examined in different HCC cell lines with Western blotting. (B) Expression of SET8 protein was examined with Western blotting in SMMC-7721 cells transfected with psi-H1-SET8 siRNA or psi-H1 plasmids. (C) CCK-8 assay showing that SET8 knockdown inhibits proliferation of SMMC-7721 cells. (D) Quantification of results from C. (E) Wound healing assay showing that SET8 knockdown suppresses migration of SMMC-7721 cells. (F) Quantification of results from E. (G) Transwell assay showing that SET8 knockdown suppresses invasion of SMMC-7721 cells. Effects of SET8 knockdown on HCC cell growth in vivo. (H) The tumour tissues were observed using an animal tissue imaging system. (I) Growth curves of xenograft tumours. (J) Effects of SET8 knockdown on chemotherapy sensitivity after treatment with cisplatin, docetaxel, etoposide, or 5-Fu in SMMC-7721 cells. Cells transfected with either SET8siRNA or control-siRNA were treated with increasing concentrations of cisplatin, docetaxel, etoposide, or 5-Fu. CCK-8 assay showing cell survival. (K) Western blot showing the protein levels of SET8, p53, and p53K382me1 upon treatment with the indicated siRNAs in SMMC-7721 cells. **p $<0.01, * \mathrm{p}<0.05$.

CCK-8 assays were performed to determine the proliferation ability of SMMC-7721 cells. SET8 knockdown markedly inhibited HCC cell proliferation from 12 to $72 \mathrm{~h}$ compared to $p s i$ - $H 1$-transfected cells and blank control cells $(\mathrm{P}<0.01$, Fig. 1C,D). Migration was assessed with the wound healing assay. Compared with SMMC7721 cells transfected with control-siRNA and blank control cells, the migration ratio was obviously decreased in SMMC-7721 cells transfected with SET8-siRNA at 12 and $24 \mathrm{~h}(\mathrm{P}<0.01$, Fig. 1E,F). In transwell assay, SET8 knockdown also dramatically inhibited SMMC-7721 cell invasion $(P<0.01$, Fig. $1 G)$. To further investigate the 
A

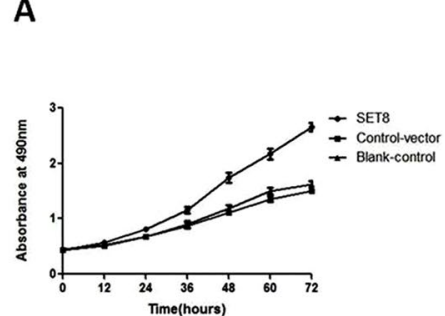

B

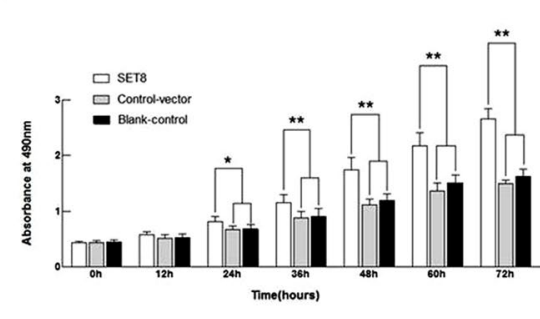

D

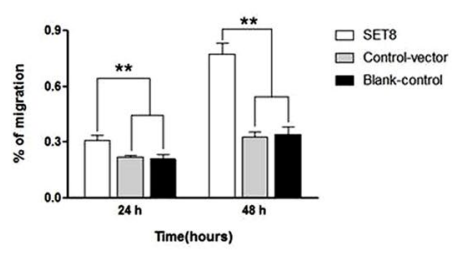

E

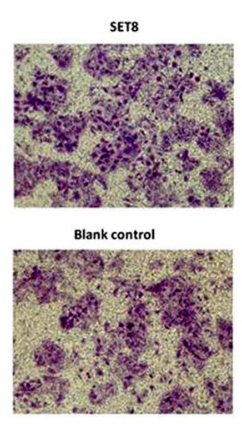

C

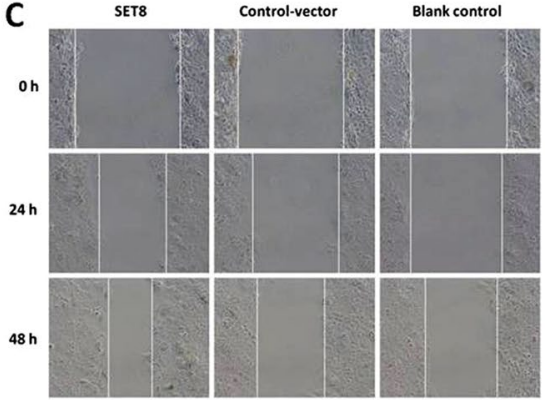

$\mathbf{F}$

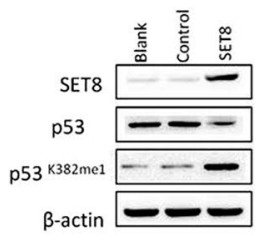

Figure 2. SET8 overexpression promotes proliferation, migration, and invasion of HCC cells. (A) CCK-8 assay showing that SET8 overexpression promotes proliferation of Huh-7 cells. (B) Quantification of results from A. (C) Wound healing assay showing that SET8 overexpression promotes migration of Huh-7 cells. (D) Quantification of results from C. (E) Transwell assay showing that SET8 overexpression stimulates invasion of Huh-7 cells. (F) Western blot showing the protein levels of SET8, p53 and p53K382me1 upon SET8 overexpression in Huh-7 cells. $* * \mathrm{p}<0.01, * \mathrm{p}<0.05$.

mechanism of cell proliferation inhibition by SET8, apoptosis was measured by Annexin V-PE/7-AAD double staining with flow cytometry. No significant difference in apoptosis was found between SET8-siRNA, control siRNA and blank control groups (data not shown). These finding suggests that SET8 knockdown inhibited proliferation, migration and invasion of SMMC-7721 cells.

While p53 expression increased, monomethylation of p53 K382 (p53K382me1) decreased upon SET8 silencing (Fig. 1K). These data demonstrated that SET8 could modulate p53 expression through methylation of K382 in HCC cells.

To further evaluate the in vivo effect of SET8 knockdown on tumour growth, the growth rate of SMMC7721 xenografts stably transfected SET8-siRNA was compared to xenografts transfected with control-siRNA. As shown in Fig. 1H,I, the growth of SET8-siRNA xenografts was significantly decreased compared with that of control-siRNA xenografts, as assessed with green fluorescent imaging. The tumour volume of SET8-siRNA xenografts was smaller than that of control-siRNA xenografts at 14, 21 and 28 days after implantation $(\mathrm{P}<0.05)$ according to calliper measurement. These results suggest that SET8 knockdown inhibited growth of SMMC-7721 cells in vivo.

Overexpression of SET8 promotes proliferation, migration and invasion of HCC cells. The effect of SET8 overexpression on HCC progression in terms of proliferation, migration and invasion was evaluated in Huh-7 cells. Cells transfected with SET8-pEZ-M61 promoted proliferation from 24 to $72 \mathrm{~h}(\mathrm{P}<0.05$, Fig. 2A,B), migration $(\mathrm{P}<0.05$, Fig. $2 \mathrm{C}, \mathrm{D})$ at 24 and $48 \mathrm{~h})$, and invasion $(\mathrm{P}<0.01$, Fig. $2 \mathrm{E})$ compared with cells transfected with pEZ-M61 or blank control cells. SET8 overexpression not only reduced p53 expression, but also increased p53K382mel (Fig. 2F). These data demonstrated that overexpression of SET8 promoted proliferation, migration and invasion of HCC cells.

An inhibitor of SET8 inhibits proliferation, migration and invasion of HCC cells. UNC0379, a selective and substrate-competitive inhibitor of SET $8^{22}$, was used to evaluate whether it mimics the effect of SET8 siRNA on HCC progression. SMMC-7721 and Huh-7 cells were treated with different concentrations of UNC0379 $(0,5$ or $10 \mu \mathrm{M})$ for $72 \mathrm{~h}$. Our results showed that growth of SMMC-7721 and Huh-7 cells was dramatically inhibited by UNC0379 (Fig. 3A,B). UNC0379 inhibited proliferation from 24 to $72 \mathrm{~h}(\mathrm{P}<0.01$, Fig. 3C,D,E,F), migration $(\mathrm{P}<0.05$, Fig. $3 \mathrm{G}, \mathrm{H}, \mathrm{I}, \mathrm{J})$ at 24 and $48 \mathrm{~h}$, and invasion $(\mathrm{P}<0.01$, Fig. $3 \mathrm{~K}, \mathrm{~L})$ in these two cell lines. This inhibitor also mimicked the effect of SET8 siRNA regarding the increase in p53 levels and the decrease in p53K382mel (Fig. 3M). These data demonstrated that SET8 was an effective target for HCC prevention. 
A

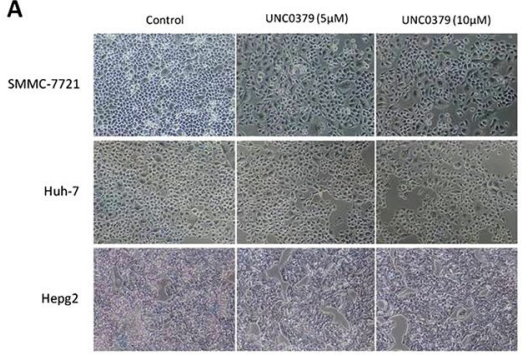

D

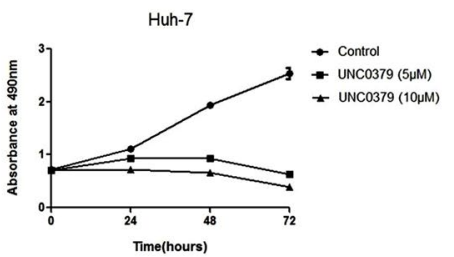

G
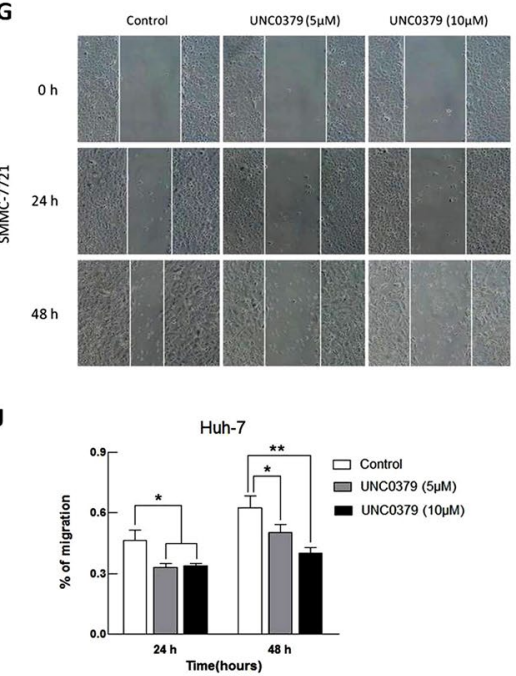

M

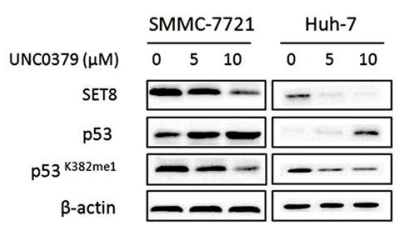

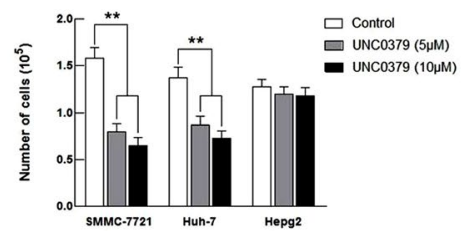

E

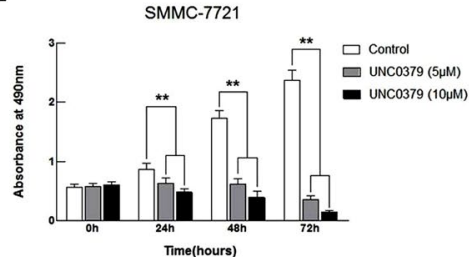

H
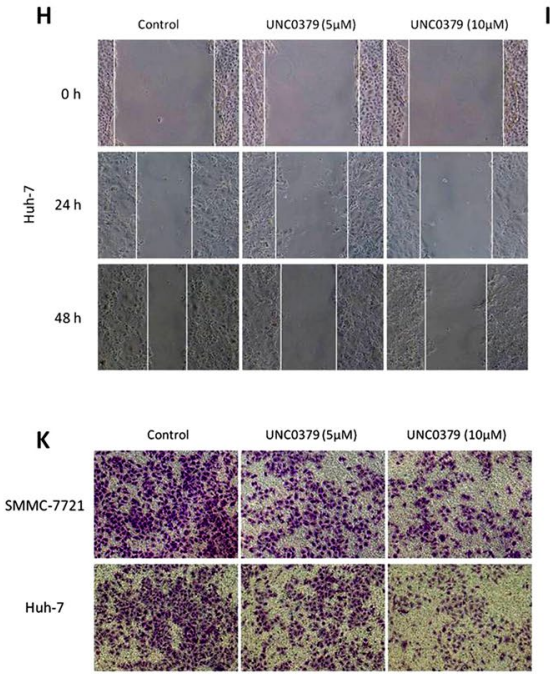
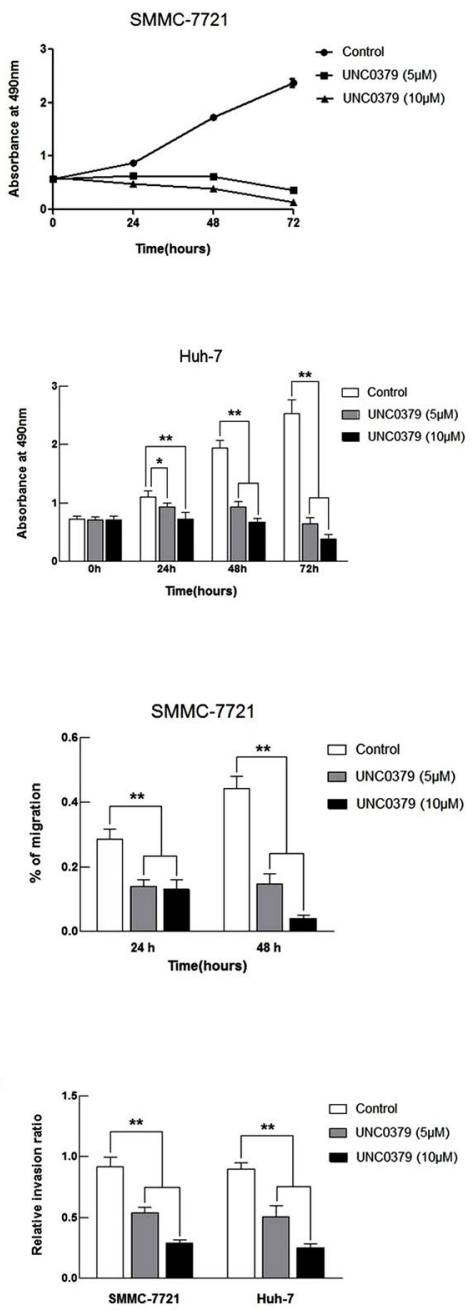

Figure 3. Effects of UNC0379 on proliferation, migration and invasion in HCC cells. (A,B) UNC0379 treatment reduces the number of HCC cells. (C,D) CCK-8 assay showing that treatment with $5 \mu \mathrm{M}$ and $10 \mu \mathrm{M}$ UNC0379 inhibits proliferation of SMMC-7721 and Huh-7 cells. (E,F) Quantification of results from C and D. $(\mathbf{G}, \mathbf{H})$ Wound healing assay showing that treatment with $5 \mu \mathrm{M}$ and $10 \mu \mathrm{M}$ UNC0379 suppresses migration of SMMC-7721 and Huh-7 cells. (I,J) Quantification of results from G and H. (K) Transwell assay showing that treatment with $5 \mu \mathrm{M}$ and $10 \mu \mathrm{M}$ UNC0379 suppresses invasion of SMMC-7721 and Huh-7 cells. (L) Quantification of results from K. (M) Western blot showing the protein levels of SET8, p53 and p53K382me1 in SMMC-7721 and Huh-7 cells treated with different concentrations of UNC0379 (5 or 10 $\mu \mathrm{M})$ for $48 \mathrm{~h}$. $* * \mathrm{p}<0.01, * \mathrm{p}<0.05$.

Knockdown of SET8 increased sensitivity of SMMC-7721 cells for docetaxel. Since we showed that SET8 may be a target for HCC prevention, we examined the synergy of SET8 knockdown with chemotherapy. We assessed whether SET8 knockdown could change chemotherapy sensitivity of SMMC-7721 cells in different concentrations of chemotherapeutic agents, such as cisplatin, docetaxel, 5-Fu and etoposide. CCK-8 assay revealed that proliferation of SMMC-7721 cells was significantly inhibited in the SET8-siRNA group after treatment with docetaxel in all doses used $(\mathrm{P}<0.01$, Fig. 1J). These results indicated that SET8 knockdown increased chemotherapy sensitivity of SMMC-7721 cells to docetaxel. 
A

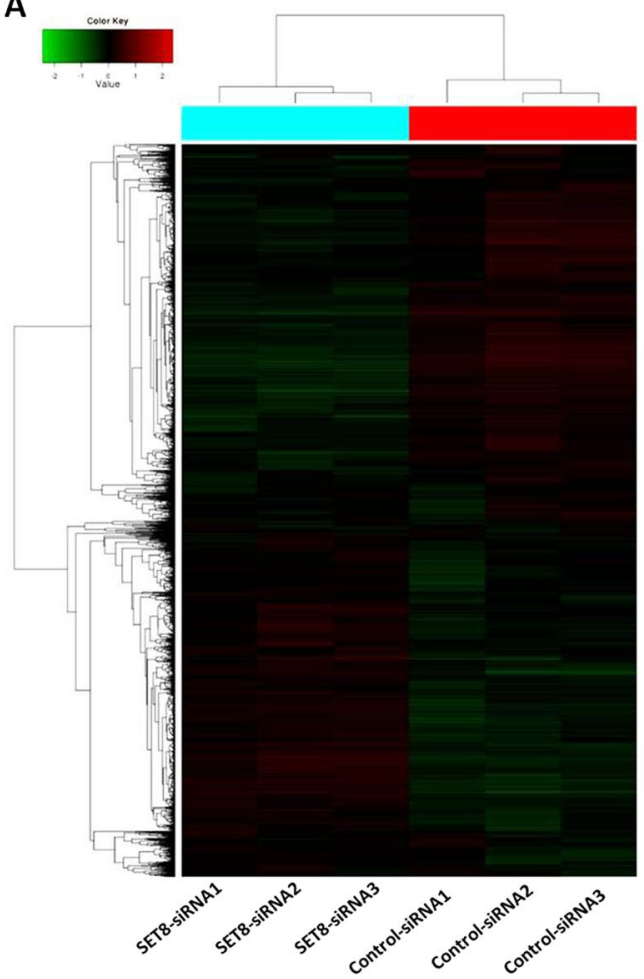

B

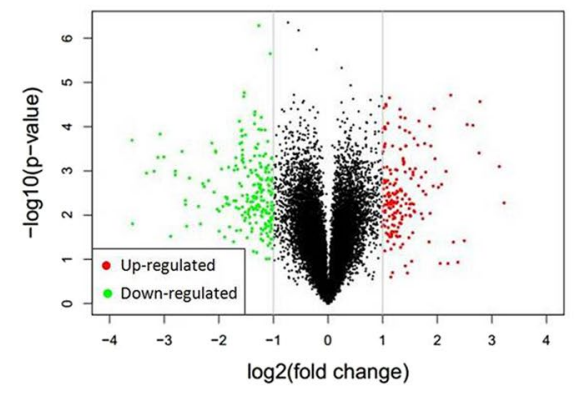

C

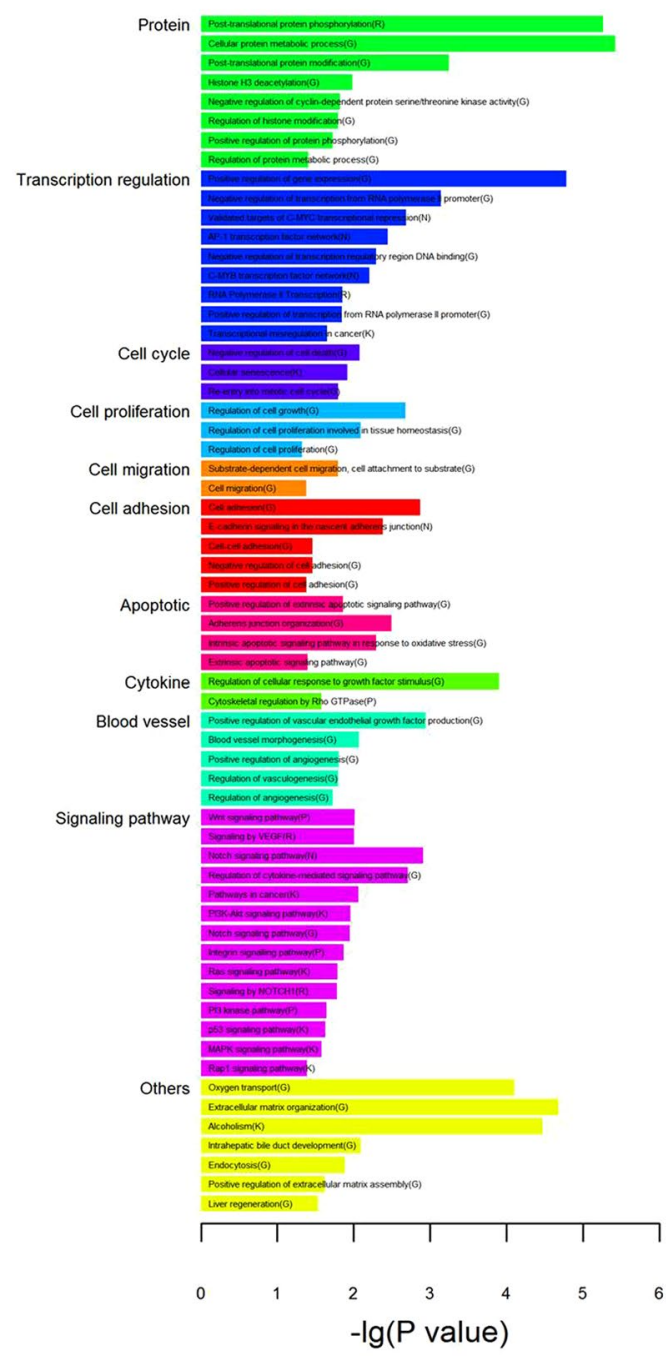

Figure 4. Gene expression profiling revealed the genome-wide influence of SET8- siRNA on SMMC-7721 cells. (A) Heat map analysis of differentially expressed genes in SET8-siRNA and control-siRNA treated cells. Up-regulated and down-regulated genes are shown in red and green, respectively. (B) Volcano plot showing the expression of genes in SET8-siRNA and control-siRNA treated cells. Red dots indicate a $>2.0$-fold change in mRNA between SET8-siRNA and control-siRNA cells. Green dots indicate a $<-2$-fold change between SET8siRNA and control-siRNA cells. (C) Reactome FI analysis with 406 genes with significantly altered expression upon SET8 knockdown. 'G' represents 'GO biological process', 'K' represents 'KEGG pathway', 'N' represents 'NCI PID', 'R' represents 'Reactome,' 'P' represents 'Reactome Pathway' and 'B' represents 'BioCarta. The detailed results are shown in Supplementary Table S2.

Gene expression profile of SET8-knockdown cells. To investigate the target genes induced by SET8 knockdown in HCC cells, we monitored the changes in mRNA levels between SET8-siRNA cells and control-siRNA cells with whole-genome expression microarray analysis (Fig. 4A). A total of 406 differentially expressed genes were identified, including 181 upregulated and 225 downregulated genes (fold change $>2.0$, $q<0.05$ ) (Fig. 4B, Supplementary Table S1). Biological network analysis for these 406 differentially expressed genes with Reactome FI showed that enrichment of biological processes related to the cell cycle, cell proliferation, cell migration, cell adhesion, apoptosis, cytokine, angiogenesis and the enriched signalling pathways, namely p53 signalling pathway ${ }^{23}$, Wnt signalling pathway ${ }^{24}$ and VEGF signalling pathway ${ }^{25}$, were associated with SET8 knockdown (Fig. 4C, Supplementary Table S2). These data demonstrated that SET8 modified the progression of HCC by modulating these signalling pathways.

\section{Discussion}

In the present study, we evaluated the function of SET8 in HCC development. We showed that SET8 knockdown inhibited proliferation of HCC cells both in vitro and in vivo. In addition, SET8 knockdown inhibited migration and invasion of HCC cells. These results are in line with previous studies that highlighted the role of SET8 in progression and metastasis of a variety of tumours, such as papillary thyroid cancer, oesophageal squamous 
cell carcinoma and renal cell carcinoma ${ }^{26-28}$. Consistent with these data, SET8 overexpression and UNC0379 treatment also proved that SET8 is an oncogene that favours HCC development. The fact that SET8 knockdown increased sensitivity of SMMC-7721 cells to docetaxel treatment implied the potential for SET8 to be used as a treatment target and biomarker for treatment efficiency. Docetaxel is a member of the taxane family that is known to disrupt microtubule formation during cell division. This drug has displayed antiproliferative activity through induction of HCC cell death in a previous study ${ }^{29}$. The potential prevention mechanism of docetaxel and its interaction with SET8 in HCC progression require further study in a larger sample size and in animal models.

A total of 406 genes were significantly altered in SET8 knockdown HCC cells, based on microarray analysis. Reactome FI analysis showed that genes altered in many biological processes referring to the cell cycle, cell proliferation, cell migration, cell adhesion, apoptosis, cytokine, angiogenesis, the Wnt signalling pathway, VEGF signalling pathway and p53 signalling pathway upon SET8 knockdown, which implied that these processes may be modified by SET8 alteration.

The Wnt signalling pathway is essential for a number of cellular functions including cell proliferation, migration and invasion ${ }^{30}$. SET8 knockdown inhibited proliferation, migration and invasion of renal carcinoma 786-O cells, potentially through Wnt/ $\beta$-catenin signalling ${ }^{28}$. Our results also demonstrated that the Wnt signalling pathway may be responsible for modulating cellular functions upon SET8 knockdown. VEGF is a major driver of angiogenesis in carcinogenesis and cancer progression that correlates with vascular and portal vein invasion and influences HCC outcome ${ }^{31}$. In addition, the novel anti-VEGF humanised monoclonal antibody BD0801 significantly inhibited proliferation of SMMC-7721, and caused cell cycle arrest at the G1 phase ${ }^{25}$. Taken together, all these signalling pathways may modify the outcome of HCC upon SET8 knockdown. Functional analysis of candidate genes should be performed in further studies.

SET8 also catalyses monomethylation of H4K20me1 and of non-histone proteins, such as p53, TWIST, Wnt, and PCNA. Accumulating evidence has shown that SET8 is crucial for gene transcription regulation during tumour formation and progression ${ }^{6,7,9}$. It has been reported that SET8 modulates p53 activity through methylation at K382 and knockdown of SET8 resulted in p53 reduction in papillary thyroid cancer cells ${ }^{26}$. We did find that SET8 modulated p53 expression through methylation of K382 in HCC cells. SET8 may influence HCC development through methylation of p53 and subsequent downstream signal changes. Additionally, SET8 induced epithelial-mesenchymal transition (EMT) through interaction with TWIST, thereby enhancing cell invasion in breast cancer ${ }^{12}$. Moreover, SET8 interacts directly with the DNA replication factor PCNA and shows specific effect at the origins of replication ${ }^{32}$. Taken together, SET8 could play an oncogenic role by facilitating tumorigenesis.

In summary, our study suggests that SET8 may modify HCC development and progression by influencing proliferation, migration and invasion through modulation of a number of genes. Therefore, SET8 may be a potential new therapeutic target for HCC treatment.

\section{Data availability}

All data generated or analysis during this study are included in this published article (and its Supplementary Information files).

Received: 4 March 2019; Accepted: 26 February 2020;

Published online: 11 March 2020

\section{References}

1. Torre, L. A. et al. Global cancer statistics, 2012. CA Cancer J Clin. 65, 87-108 (2015).

2. Choi, K. J., Baik, I. H., Ye, S. K. \& Lee, Y. H. Molecular targeted therapy for hepatocellular carcinoma: present status and future directions. Biol Pharm Bull. 38, 986-991 (2015).

3. Beck, D. B., Oda, H., Shen, S. S. \& Reinberg, D. PR-Set7 and H4K20me1: at the crossroads of genome integrity, cell cycle, chromosome condensation, and transcription. Genes Dev. 26, 325-337 (2012).

4. Liu, B. et al. A functional single nucleotide polymorphism of SET8 is prognostic for breast cancer. Oncotarget. 7, 34277-34287 (2016).

5. Weirich, S., Kusevic, D., Kudithipudi, S. \& Jeltsch, A. Investigation of the methylation of Numb by the SET8 protein lysine methyltransferase. Sci Rep. 5, 13813 (2015).

6. Tardat, M., Murr, R., Herceg, Z., Sardet, C. \& Julien, E. PR-Set7-dependent lysine methylation ensures genome replication and stability through S phase. J. Cell Biol. 179, 1413-1426 (2007).

7. Jørgensen, S. et al. The histone methyltransferase SET8 is required for S-phase progression. J. Cell Biol. 179, 1337-1345 (2007).

8. Abbas, T. et al. CRL4(Cdt2) regulates cell proliferation and histone gene expression by targeting PR-Set7/Set8 for degradation. Mol Cell. 40, 9-21 (2010).

9. Huen, M. S., Sy, S. M., van Deursen, J. M. \& Chen, J. Direct interaction between SET8 and proliferating cell nuclear antigen couples H4-K20 methylation with DNA replication. J. Biol Chem. 283, 11073-11077 (2008).

10. Schotta, G. et al. A chromatin-wide transition to H4K20 monomethylation impairs genome integrity and programmed DNA rearrangements in the mouse. Genes Dev. 22, 2048-2061 (2008).

11. Shi, X. et al. Modulation of p53 function by SET8-mediated methylation at lysine 382. Mol Cell. 27, 636-646 (2007)

12. Yang, F. et al. SET8 promotes epithelial-mesenchymal transition and confers TWIST dual transcriptional activities. EMBO J. 31, 110-123 (2012).

13. Li, Z., Nie, F., Wang, S. \& Li, L. Histone H4 Lys 20 monomethylation by histone methylase SET8 mediates Wnt target gene activation. Proc. Natl Acad Sci. USA 108, 3116-3123 (2011).

14. Li, Y. et al. The histone modifications governing TFF1 transcription mediated by estrogen receptor. J. Biol Chem. 286, 13925-13936 (2011).

15. Dulev, S., Tkach, J., Lin, S. \& Batada, N. N. SET8 methyltransferase activity during the DNA double- strand break response is required for recruitment of 53BP1. EMBO Rep. 15, 1163-1174 (2014).

16. Guo, Z. et al. A polymorphism at the miR-502 binding site in the 3'-untranslated region of the histone methyltransferase SET8 is associated with hepatocellular carcinoma outcome. Int J. Cancer. 131, 1318-1322 (2012). 
17. Cui, N., Yang, W. T. \& Zheng, P. S. Slug inhibits the proliferation and tumor formation of human cervical cancer cells by upregulating the $\mathrm{p} 21 / \mathrm{p} 27$ proteins and down-regulating the activity of the Wnt/beta-catenin signaling pathway via the transsuppression Akt1/p-Akt1 expression. Oncotarget. 7, 26152-26167 (2016).

18. Meng, F. et al. MiR-30a-5p Overexpression may overcome EGFR-inhibitor resistance through regulating PI3K/AKT Signaling pathway in non-small cell lung cancer cell lines. Front Genet. 7, 197 (2016).

19. National Research Council of the National Academies: Guide for the care and use of laboratory animals. 8th edition. The National Academies Press, Washington, D.C., USA. pp199-200, 2011.

20. Zhou, M. et al. Toxicogenomic analysis identifies the apoptotic pathway as the main cause of hepatotoxicity induced by tributyltin. Food Chem Toxicol. 97, 316-326 (2016).

21. Croft, D. et al. Reactome: a database of reactions, pathways and biological processes. Nucleic Acids Res. 39, D691-697 (2011).

22. Ma, A. et al. Discovery of a selective, substrate-competitive inhibitor of the lysine methyltransferase SETD8. J Med Chem. 57, 6822-6833 (2014).

23. Meng, X., Franklin, D. A., Dong, J. \& Zhang, Y. MDM2-p53 pathway in hepatocellular carcinoma. Cancer Res. 74, 7161-7167 (2014),

24. Kim, W. et al. Hippo signaling interactions with Wnt/beta-catenin and Notch signaling repress liver tumorigenesis. J. Clin Invest. 127, 137-152 (2017).

25. Liu, L. et al. Molecular targeting of VEGF/VEGFR signaling by the anti-VEGF monoclonal antibody BD0801 inhibits the growth and induces apoptosis of human hepatocellular carcinoma cells in vitro and in vivo. Cancer Biol Ther. 18, 166-176 (2017).

26. Liao, T. et al. Histone methyltransferase KMT5A gene modulates oncogenesis and lipid metabolism of papillary thyroid cancer in vitro. Oncol Rep. 39, 2185-2192 (2018).

27. Wang, C., Wu, J., Zhao, Y. \& Guo, Z. miR-502 medaited histone methyltransferase SET8 expression is associated with outcome of esophageal squamous cell carcinoma. Sci Rep. 6, 32921 (2016).

28. Zhang, S. et al. miR-502-mediated histone methyltransferase SET8 expression is associated with clear cell renal cell carcinoma risk. Oncol Lett. 14, 7131-7138 (2017).

29. Chunhu, Z., Suiyu, H., Meiqun, C., Guilin, X. \& Yunhui, L. Antiproliferative and apoptotic effects of paeonol on human hepatocellular carcinoma cells. Anticancer Drugs. 19, 401-409 (2008).

30. Clevers, H. \& Nusse, R. Wnt/beta-catenin signaling and disease. Cell. 149, 1192-1205 (2012).

31. Poon, R. T. et al. Serum vascular endothelial growth factor predicts venous invasion in hepatocellular carcinoma: A Prospective Study. Ann Surg. 233, 227-235 (2001).

32. Takawa, M. et al. Histone lysine methyltransferase SETD8 promotes carcinogenesis by deregulating PCNA expression. Cancer Res. 72, 3217-3227 (2012)

\section{Acknowledgements}

The present study was supported by the Natural Science Foundation of China of Hebei Province (Grant No. H2019206428) and the Science and Technology Project of Hebei (Grant No. 162777114D).

\section{Author contributions}

J.W. and K.Q. performed all experiments, Y.D. collected data, X.Z. and H.C. performed statistical analysis, Z.G. and L.P. designed the experiments, conceived the concept, and wrote the manuscript. All authors were involved in writing the paper and had final approval of the submitted and published versions.

\section{Competing interests}

The authors declare no competing interests.

\section{Additional information}

Supplementary information is available for this paper at https://doi.org/10.1038/s41598-020-61402-7.

Correspondence and requests for materials should be addressed to L.P. or Z.G.

Reprints and permissions information is available at www.nature.com/reprints.

Publisher's note Springer Nature remains neutral with regard to jurisdictional claims in published maps and institutional affiliations.

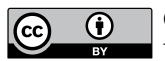

Open Access This article is licensed under a Creative Commons Attribution 4.0 International License, which permits use, sharing, adaptation, distribution and reproduction in any medium or format, as long as you give appropriate credit to the original author(s) and the source, provide a link to the Creative Commons license, and indicate if changes were made. The images or other third party material in this article are included in the article's Creative Commons license, unless indicated otherwise in a credit line to the material. If material is not included in the article's Creative Commons license and your intended use is not permitted by statutory regulation or exceeds the permitted use, you will need to obtain permission directly from the copyright holder. To view a copy of this license, visit http://creativecommons.org/licenses/by/4.0/.

(C) The Author(s) 2020 